\title{
Compensação de EDPs da onda na dinâmica de atuação para busca extremal
}

\author{
Tiago Roux Oliveira* Pedro Zanetti de Albuquerque* \\ * Programa de Pós-Graduação em Engenharia Eletrônica (PEL), \\ Universidade do Estado do Rio de Janeiro (UERJ), Rua São Francisco \\ Xavier, 524 - Maracanã, Rio de Janeiro, RJ, Brasil. \\ (e-mail: tiagoroux@uerj.br,pedro.zanetti.albuquerque@gmail.com)
}

\begin{abstract}
Gradient extremum seeking for compensating wave actuator dynamics in cascade with static scalar maps is addressed in the present paper. This class of Partial Differential Equations (PDEs) for extremum seeking has not been studied yet. A dynamic feedback control law based on distributed parameters is proposed by employing backstepping transformation with an appropriate target system and an adequate formulation using Neumann interconnections. Local stability and convergence to a small neighborhood of the desired (but unknown) extremum is proved by means of a Lyapunov functional and the theory of averaging in infinite dimensions. Numerical simulations illustrate the theoretical results.

Resumo: Neste artigo é apresentado um controlador por busca extremal baseado no método gradiente para compensar a dinâmica de atuação com dimensão infinita do tipo equação da onda em cascata com um mapeamento escalar estático desconhecido. Esta classe de Equações Diferenciais Parciais (EDPs) ainda não havia sido estudada para busca extremal. Uma lei de controle dinâmica baseada em parâmetros distribuídos é proposta fazendo uso da transformação backstepping com sistema alvo apropriado e adequada formulação usando interconexões de Neumann. A estabilidade local e a convergência para uma pequena vizinhança do extremo (desconhecido) desejado são provadas por meio de um funcional de Lyapunov e a teoria da média em dimensões infinitas. Simulações numéricas ilustram os resultados teóricos.
\end{abstract}

Keywords: Extremum seeking; Adaptive control; Backstepping; Averaging theory; Infinite-dimensional systems; Partial differential equations.

Palavras-chaves: Busca extremal; Controle adaptativo; Backstepping; Teoria da média; Sistemas de dimensão infinita; Equações diferenciais parciais.

\section{INTRODUÇÃO}

A Busca Extremal tem recebido grande atenção na comunidade de controle, sendo reconhecida como uma das poderosas metodologias em sistemas adaptativos para enfrentar problemas de controle onde a planta é mal modelada ou seu modelo é contaminado por incertezas severas e dinâmica não modelada (Krstić and Wang (2000)).

Embora a busca extremal tenha sido empregada com sucesso em muitas aplicações de engenharia, os autores em (Oliveira et al. (2017), Rusiti et al. (2018) e Rusiti et al. (2019)) apontam a presença de atraso como um fator limitante na aplicação do método em situações práticas. Estas publicações proveram uma análise detalhada e completa da aplicação de projetos para compensação de atrasos em busca extremal com mapeamentos estáticos e dinâmicos. Isto fornece um sistemático e efetivo projeto para os algoritmos baseados em gradiente e Newton. Em particular, apenas Equações Diferenciais Parciais (EDPs) hiperbólicas de transporte de primeira ordem são originalmente consideradas em (Oliveira et al. (2017)) para representar atrasos. Esta ideia chave tem permitido o desenvolvimento de extensões para outras classes de EDPs, tais como as descritas fenômeno de difusão estudadas em (Feiling et al. (2018) e Oliveira et al. (2018)).

\footnotetext{
ऋ Este trabalho foi financiado por CNPq, CAPES e FAPERJ no Brasil.
}

Neste trabalho, expandimos a classe de EDPs para qual a Busca Extremal pode ser aplicada, considerando a dinâmica da onda em cascata com um mapeamento escalar estático a ser otimizado. O problema que enfrentamos no presente artigo pode ser inspirado por aplicações de engenharia específicas relacionadas com perfuração off-shore (Bekiaris-Liberis and Krstić (2014)) ou até controle óptico (Aarsnes et al. (2019)), onde a abordagem de otimização em tempo real seria afetada por uma EDP de onda na dinâmica de atuação. A equação de onda é desafiadora devido ao fato de que todos os seus autovalores (infinitamente muitos) estão no eixo imaginário, e devido ao fato de que ela tem uma velocidade de propagação finita (limitada) (Krstić (2009)).

Nosso trabalho é a primeira contribuição de aplicação da busca extremal em uma dinâmica de atuação de dimensão infinita governada por EDPs de onda. O problema estudado aqui é mais desafiador do que o caso de difusão em (Feiling et al. (2018), Oliveira et al. (2018)) devido a outra dificuldade - o sistema de EDP é de segunda ordem no tempo, o que significa que o estado é "duplamente infinito dimensional" (deslocamento distribuído e velocidade distribuída). Isto não é tanto um problema dimensional, como é um problema na construção das transformações de estado para compensar a dinâmica da EDP (Krstić (2009)). É preciso lidar com o acoplamento de dois estados de dimensão infinita. 
O projeto de controle completo empregando um compensador para a dinâmica de atuação da onda é desenvolvido via transformação de backstepping, retornando as estimativas para o gradiente e Hessian (primeira e segunda derivadas) do mapa estático a ser maximizado. Nossas provas para a estabilidade local do sistema em malha fechada e a convergência para uma pequena vizinhança do extremo são baseadas na metodologia de backstepping para controle da EDP (Krstić and Smyshlyaev (2008)), na construção de um funcional de Lyapunov e no uso do teorema de média para sistemas infinito dimensionais (Hale and Lunel (1990)). Para o melhor de nosso conhecimento, todos esses resultados de sistemas de parâmetros distribuídos para busca extremal com compensação de onda são novos.

\subsection{Notação e terminologia}

Nós denotamos as derivadas parciais de uma função $u(x, t)$ como $u_{x}(x, t):=\partial_{x} u(x, t)=\partial u(x, t) / \partial x, u_{t}(x, t):=\partial_{t} u(x, t)=$ $\partial u(x, t) / \partial t$, ou ainda $u_{x x}(x, t):=\partial_{x x} u(x, t)$ e $u_{t t}(x, t):=\partial_{t t} u(x, t)$ para as derivadas parciais de segunda ordem com respeito a $x$ e $t$, respectivamente. A norma-2 (Euclidiana) de um vetor de estado $\vartheta(t)$ de dimensão finita de uma Equação Diferencial Ordinária (EDO) é denotada por barras simples, $|\vartheta(t)|$. Em contraste, normas de funções (de x) são denotadas por barras duplas. Nós denotamos a norma espacial $\mathscr{L}_{2}[0, D]$ do estado $u(x, t)$ de uma EDP como $\|u(t)\|_{\mathscr{L}_{2}([0, D])}^{2}:=\int_{0}^{D} u^{2}(x, t) d x$, onde, na sequencia, nós descartamos o índice $\mathscr{L}_{2}([0, D])$, portanto, $\|$. $\|=\| \cdot \|_{\mathscr{L}_{2}([0, D])}$, se não for especificado de outra forma. Como definido em (Khalil (1996)), um vetor função $f(t, \varepsilon) \in \mathbb{R}^{n}$ é dito ser de ordem $\mathscr{O}(\varepsilon)$ em um intervalo $\left[t_{1}, t_{2}\right]$, se $\exists k, \bar{\varepsilon}$ : $|f(t, \varepsilon)| \leq k \varepsilon, \forall \varepsilon \in[0, \bar{\varepsilon}]$ e $\forall t \in\left[t_{1}, t_{2}\right]$. Na maioria dos casos nós não damos estimativa da constante $k$ e $\bar{\varepsilon}$, então $\mathscr{O}(\varepsilon)$ pode ser interpretada como uma relação de ordem de magnitude para $\varepsilon$ suficientemente pequeno.

\section{FORMULAÇÃO DO PROBLEMA}

\subsection{Busca Extremal Básica para Mapeamentos Estáticos}

No mais simples caso da busca extremal para mapeamentos estáticos, o objetivo é encontrar e manter o ótimo de um mapeamento estático não linear desconhecido $Q(\cdot)$ com saída ótima desconhecida $y^{*}$, otimizador desconhecido $\theta^{*}$, saída mensurável $y$ e entrada $\theta$. Sem perda de generalidade, consideramos o problema de maximação.

O método de perturbação senoidal (Krstić and Wang (2000)) varia o parâmetro de entrada $\theta$ do mapeamento estático para obter uma estimativa do gradiente $G$ para o mapeamento estático. Portanto, o sinal aditivo de perturbação

$$
S(t)=a \sin (\omega t)
$$

com amplitude $a$ e frequência $\omega$, é adicionado a estimação do otimizador $\theta^{*}$, dado por $\hat{\theta}$. O sinal de perturbação multiplicativo, para estimar o gradiente do mapeamento estático, é escolhido como

$$
M(t)=\frac{2}{a} \sin (\omega t)
$$

A idéia de escolher os sinais de perturbação como (1) e (2) assim como a lei de adaptação $\dot{\hat{\theta}}(t)=k G(t)$ é alcançar o sinal médio do gradiente estimado $G$ dado por $G_{\mathrm{av}}(t)=H \tilde{\theta}_{\mathrm{av}}=$ $H\left(\hat{\theta}_{\mathrm{av}}-\theta^{*}\right)$, onde $H$ é a Hessiana desconhecida negativa do mapeamento estático e $\tilde{\theta}=\hat{\theta}-\theta^{*}$ é a estimação do erro. Isso

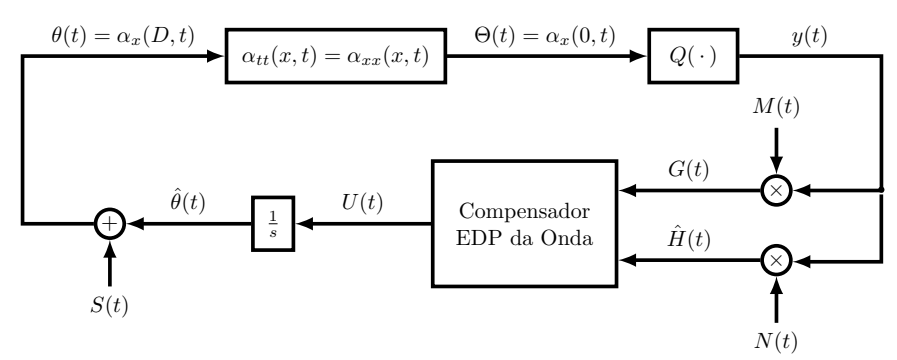

Figura 1. Busca Extremal baseada no método de gradiente com dinâmica de atuação governada por EDP da onda com controlador conpensando (40), sinais de perturbação aditivo e multiplicativo (2), (24) e (26), respectivamente.

resulta na dinâmica média do erro $\dot{\tilde{\theta}}_{\mathrm{av}}=K H \tilde{\theta}_{\mathrm{av}}$, com ganho de adaptação $K>0$. O sistema médio é exponencialmente estável e pelo teorema da média em (Khalil (1996)), a dinâmica de erro original é exponencial estável em relação a um pequeno conjunto residual.

\subsection{Dinâmica de atuação da onda e sinal de saída}

Consideramos dinâmicas de atuação que são descritas por um processo de onda, ou seja, uma EDP da onda com o atuador $\theta(t)$ e o atuador propagado $\Theta(t)$ dados por

$$
\begin{aligned}
& \Theta(t)=\partial_{x} \alpha(0, t) \\
& \partial_{t t} \alpha(x, t)=\partial_{x x} \alpha(x, t), \quad x \in[0, D] \\
& \alpha(0, t)=0 \\
& \partial_{x} \alpha(D, t)=\theta(t),
\end{aligned}
$$

onde o comprimento do domínio $D$ é conhecido. A medição é definida pelo mapeamento estático desconhecido com entrada (3), tal que

$$
y(t)=Q(\Theta(t)) .
$$

Por uma questão de simplicidade, assumimos o seguinte:

Assumption 1. O mapa estático não linear desconhecido é quadrático, ou seja,

$$
Q(\Theta)=y^{*}+\frac{H}{2}\left(\Theta-\Theta^{*}\right)^{2},
$$

onde além de $\Theta^{*} \in \mathbb{R}$ e $y^{*} \in \mathbb{R}$ serem desconhecidos, o escalar $H<0$ é a Hessiana desconhecida do mapeamento estático.

Assim, a saída do mapeamento estático é dada por

$$
y(t)=y^{*}+\frac{H}{2}\left(\Theta(t)-\Theta^{*}\right)^{2} .
$$

Combinando a dinâmica de atuação acima e o esquema básico da busca extremal, adaptando ainda mais o esquema proposto em (Oliveira et al. (2017)), o sistema em malha fechada de busca extremal com dinâmica de atuação governada por uma EDP da onda sob um controlador de compensação da onda desconhecido é mostrado na Figura 1.

\subsection{Sinais e sistemas}

Como no esquema básico da busca extremal, definimos a entrada ótima desconhecida $\theta^{*}$ de $\theta(t)$ com respeito ao mapeamento estático e o processo da onda, com a relação $\Theta^{*}=\theta^{*}$. Como nosso objetivo é determinar a entrada ótima desconhecida $\theta^{*}$, definimos os erros de estimação

$$
\tilde{\theta}(t):=\hat{\theta}(t)-\theta^{*},
$$


onde $\hat{\theta}(t)$ é a estimação de $\theta^{*}$. Para que (10) seja consistente com o otimizador do mapeamento estático $\Theta^{*}$, introduzimos o erro de estimação propagado $\vartheta(t):=\hat{\Theta}(t)-\Theta^{*}$ pelo domínio da EDP da onda

$$
\begin{aligned}
& \vartheta(t):=\partial_{x} \bar{\alpha}(0, t) \\
& \partial_{t t} \bar{\alpha}(x, t)=\partial_{x x} \bar{\alpha}(x, t), \quad x \in[0, D] \\
& \bar{\alpha}(0, t)=0 \\
& \partial_{x} \bar{\alpha}(D, t)=\tilde{\theta}(t) .
\end{aligned}
$$

Da malha de controle na Figura 1 temos

$$
\dot{\hat{\theta}}(t)=U(t) \text {. }
$$

Tomando a derivada do tempo de (11)-(14) e com a ajuda de (10) e (15), a dinâmica do erro propagado é escrita como a seguinte cascata de uma EDP da onda e uma EDO (integrador) com a interconexão de Neumann (Susto and Krstić (2010)):

$$
\begin{aligned}
& \dot{\vartheta}(t)=\partial_{x} u(0, t), \\
& \partial_{t t} u(x, t)=\partial_{x x} u(x, t), \quad x \in[0, D] \\
& u(0, t)=0, \\
& \partial_{x} u(D, t)=U(t),
\end{aligned}
$$

onde $\dot{\tilde{\theta}}(t)=\dot{\hat{\theta}}(t)$, uma vez que $\theta^{*}$ é constante. Assim como na busca extremal sem atuação através do domínio da EDP da onda, o sinal de perturbação $S(t)$ deve somar $a \sin (\omega t)$ a $\Theta(t)$, assim compensa o processo de onda. Consequentemente, $a \sin (\omega t)$ com amplitude de perturbação $a$ e frequência $\omega$ é aplicado como segue:

$$
\begin{aligned}
& S(t):=\partial_{x} \beta(D, t) \\
& \partial_{t t} \beta(x, t)=\partial_{x x} \beta(x, t), \quad x \in[0, D] \\
& \beta(0, t)=0 \\
& \partial_{x} \beta(0, t)=a \sin (\omega t) .
\end{aligned}
$$

As equações (20)-(23) descrevem um problema de geração de trajetória como em (Krstić and Smyshlyaev, 2008, Chapter 12). A solução explicita de (20) é dada por

$$
S(t)=\frac{a}{\omega} \sin (\omega D) \sin (\omega t)
$$

A relação entre o erro de estimativa propagado $\vartheta(t)$, a entrada propagada $\Theta(t)$, e o otimizador do mapeamento estático $\Theta^{*}$ é dada por

$$
\vartheta(t)+a \sin (\omega t)=\Theta(t)-\Theta^{*}
$$

que pode ser facilmente comprovado, considerando $\theta(t)=$ $\hat{\theta}(t)+S(t)$ junto com as soluções de (3)-(6), (11)-(14) e (20)(23). Nos resta definir o sinal de perturbação $N(t)$ que é usado para estimar a Hessiana do mapeamento estático multiplicandoo com a saída $y(t)$ do mesmo. Em (Ghaffari et al. (2012)), a estimativa da Hessiana é derivada como

$$
\hat{H}(t)=N(t) y(t) \quad \text { com } \quad N(t)=-\frac{8}{a^{2}} \cos (2 \omega t) .
$$

Note que o sinal de perturbação $M(t)$, para estimar o gradiente, é o mesmo que no esquema básico da busca extremal (veja (2)), tal que

$$
G(t)=M(t) y(t)
$$

\section{PROJETO DO CONTROLADOR E SISTEMA EM MALHA FECHADA}

Nesta seção, apresentamos o projeto do controlador proposto, com estimativas baseadas nos sinais de perturbação (baseadas na média) do gradiente e da Hessiana, usado para compensação da onda na malha de realimentação do controlador por busca extremal da Figura 1.

\subsection{Compensação da EDP da onda via estimativa da Hessiana}

Consideramos a cascata EDP-EDO (16)-(19). Como em (Susto and Krstić (2010)), usamos a transformação backstepping

$$
w(x, t)=u(x, t)-\int_{0}^{x} l(x, \sigma) u_{t}(\sigma, t) d \sigma-\gamma(x) \vartheta(t),
$$

com ganho

$$
\begin{aligned}
l(x, \sigma) & =\gamma(x-\sigma) \\
\gamma(x) & =\bar{K}\left[\begin{array}{ll}
0 & I
\end{array}\right] e^{A x}\left[\begin{array}{ll}
I & 0
\end{array}\right]^{T}, \quad A=\left(\begin{array}{ll}
0 & 0 \\
I & 0
\end{array}\right),
\end{aligned}
$$

que transforma (16)-(19) no sistema alvo

$$
\begin{aligned}
& \dot{\vartheta}(t)=\bar{K} \vartheta(t)+w_{x}(0, t), \quad \bar{K}<0, \\
& \partial_{t t} w(x, t)=\partial_{x x} w(x, t), \quad x \in[0, D], \\
& w(0, t)=0, \\
& w_{x}(D, t)=-\bar{c} w_{t}(D, t), \quad \bar{c}>0 .
\end{aligned}
$$

Uma vez que o sistema alvo (31)-(34) é exponencialmente estável, o controlador que compensa o processo da onda pode ser obtido avaliando-se a transformação backstepping (28) em $x=D$ como

$$
\begin{aligned}
U(t) & =\bar{c}\left[\bar{K} u(D, t)-\partial_{t} u(D, t)\right]+\rho(D) \vartheta(t) \\
& +\int_{0}^{D} \rho(D-\sigma) \partial_{t} u(\sigma, t) d \sigma,
\end{aligned}
$$

onde $\rho(s)=\bar{K}\left[\begin{array}{ll}0 & I\end{array}\right] e^{A s}\left[\begin{array}{ll}0 & I\end{array}\right]^{T}$. No entanto, a lei de controle proposta em (35) não é diretamente aplicável, por que não temos medição em $\vartheta(t)$. Assim, introduzimos um resultado importante de (Ghaffari et al. (2012)): a versão média do gradiente (27) e da estimativa da Hessiana (26) são

$$
G_{\mathrm{av}}(t)=H \vartheta_{\mathrm{av}}(t), \quad \hat{H}_{\mathrm{av}}(t)=H,
$$

se um mapeamento quadrático como em (8) for considerado. Para prova de (36), veja (Ghaffari et al. (2012)). A respeito de (36), tiramos a média de (35) e escolhemos $\bar{K}=K H \operatorname{com} K>0$, tal que

$$
\begin{aligned}
U_{\mathrm{av}}(t) & =\bar{c}\left[K H u_{\mathrm{av}}(D, t)-\partial_{t} u_{\mathrm{av}}(D, t)\right]+\bar{\rho}(D) K H \vartheta_{\mathrm{av}}(t) \\
& +K H \int_{0}^{D} \bar{\rho}(D-\sigma) \partial_{t} u_{\mathrm{av}}(\sigma, t) d \sigma
\end{aligned}
$$

com

$$
\bar{\rho}(s)=\left[\begin{array}{ll}
0 & I
\end{array}\right] e^{A s}\left[\begin{array}{ll}
0 & I
\end{array}\right]^{T}, \quad A=\left(\begin{array}{ll}
0 & 0 \\
I & 0
\end{array}\right) .
$$

Ao substituir a estimativa da média (36) em (37), obtemos

$$
\begin{aligned}
U_{\mathrm{av}}(t) & =\bar{c}\left[K H u_{\mathrm{av}}(D, t)-\partial_{t} u_{\mathrm{av}}(D, t)\right]+\bar{\rho}(D) K G_{\mathrm{av}}(t) \\
& +K H \int_{0}^{D} \bar{\rho}(D-\sigma) \partial_{t} u_{\mathrm{av}}(\sigma, t) d \sigma .
\end{aligned}
$$

Devido a razões técnicas na aplicação do teorema da média para sistemas de dimensão infinita (Hale and Lunel (1990)) na seguinte prova de estabilidade, introduzimos um filtro passa baixa ao controlador. Finalmente, obtemos a lei de controle de dimensão infinita baseada em média para compensar o processo de onda:

$$
\begin{aligned}
U(t) & =\frac{c}{s+c}\left\{\bar{c}\left[K \hat{H}(t) u(D, t)-\partial_{t} u(D, t)\right]+\bar{\rho}(D) K G(t)\right. \\
& \left.+K \hat{H}(t) \int_{0}^{D} \bar{\rho}(D-\sigma) \partial_{t} u(\sigma, t) d \sigma\right\},
\end{aligned}
$$

onde $c>0$ é escolhido depois. Por conveniência de notação, misturamos o domínio do tempo e da frequência em (40), em que o filtro passa-baixa atua como um operador no termo entre chaves. 


\subsection{Sistema em malha fechada}

Substituindo (40) em (19), podemos escrever o sistema em malha fechada (16)-(19) como

$$
\begin{aligned}
& \dot{\vartheta}(t)=\partial_{x} u(0, t), \\
& \partial_{t t} u(x, t)=\partial_{x x} u(x, t), \quad x \in[0, D], \\
& u(0, t)=0, \\
& \partial_{x} u(D, t)=\frac{c}{s+c}\left\{\bar{c}\left[K \hat{H}(t) u(D, t)-\partial_{t} u(D, t)\right]+\bar{\rho}(D) K G(t)\right. \\
& \left.+K \hat{H}(t) \int_{0}^{D} \bar{\rho}(D-\sigma) \partial_{t} u(\sigma, t) d \sigma\right\} .
\end{aligned}
$$

\section{ANÁLISE DE ESTABILIDADE}

A existência de um funcional de Lyapunov através de transformação backstepping (Krstić and Smyshlyaev (2008)) permite a análise de estabilidade do sistema de realimentação completo com uma representação em cascata das equações EDP-EDO e a lei de controle de dimensão infinita.

O teorema a seguir resume as propriedades de estabilidade e convergência local do sistema em malha fechada.

Teorema 2. Considere o sistema na Figura 1 com o sistema dinâmico sendo representado pelo mapeamento quadrático não linear em (9) em cascata com a dinâmica de atuação governada pela EDP da onda em (3)-(6). Para $c>0$ suficientemente grande, existe um $\bar{\omega}(c)>0$, tal que $\forall \omega>\bar{\omega}$, o sistema em malha fechada (41)-(44) com estados $\vartheta(t), u(x, t)$, tem uma solução periódica única localmente exponencialmente estável em $t$ de período $\Pi:=2 \pi / \omega$, denotado por $\vartheta^{\Pi}(t), u^{\Pi}(x, t)$, satisfazendo $\forall t \geq 0$ :

$$
\begin{aligned}
\left(\left|\vartheta^{\Pi}(t)\right|^{2}+\left\|\partial_{x} u^{\Pi}(t)\right\|^{2}+\left\|\partial_{t} u^{\Pi}(t)\right\|^{2}\right. & \\
\left.+\left|\partial_{x} u^{\Pi}(D, t)\right|^{2}\right)^{1 / 2} & \leq \mathscr{O}(1 / \omega) .
\end{aligned}
$$

Além disso,

$$
\begin{aligned}
\limsup _{t \rightarrow \infty}\left|\theta(t)-\theta^{*}\right| & =\mathscr{O}(a / \omega+1 / \omega), \\
\limsup _{t \rightarrow \infty}\left|\Theta(t)-\Theta^{*}\right| & =\mathscr{O}(a+1 / \omega), \\
\limsup _{t \rightarrow \infty}\left|y(t)-y^{*}\right| & =\mathscr{O}\left(a+1 / \omega^{2}\right) .
\end{aligned}
$$

Prova: A prova está estruturada nos Passos 1 ao 6, analogamente ao que foi feito na referência (Feiling et al. (2018)).

Primeiramente, no Passos 1-4, mostramos a estabilidade exponencial do sistema médio em malha fechada de (41)-(44) através da transformação backstepping. Então, o teorema da média para sistemas de dimensão infinita ((Hale and Lunel, 1990, Section 2)) é invocado no Passo 5 para mostrar a estabilidade exponencial do sistema em malha fechada original (41)-(44). Finalmente, o Passo 6 mostra a convergência de $(\theta(t), \Theta(t), y(t))$ para uma pequena vizinhança do extremo $\left(\theta^{*}, \Theta^{*}, y^{*}\right)$.

\section{Passo 1: Sistema em malha fechada médio}

A versão média do sistema (41)-(44) para $\omega$ grande é

$$
\begin{aligned}
& \dot{\vartheta}_{\mathrm{av}}(t)=\partial_{x} u_{\mathrm{av}}(0, t), \\
& \partial_{t t} u_{\mathrm{av}}(x, t)=\partial_{x x} u_{\mathrm{av}}(x, t), \quad x \in[0, D] \\
& u_{\mathrm{av}}(0, t)=0 \\
& \frac{d}{d t} \partial_{x} u_{\mathrm{av}}(D, t)=-c \partial_{x} u_{\mathrm{av}}(D, t) \\
& -c\left[\bar{c}\left[K H u_{\mathrm{av}}(D, t)-\partial_{t} u_{\mathrm{av}}(D, t)\right]+\bar{\rho}(D) K H \vartheta_{\mathrm{av}}(t)\right. \\
& \left.\quad+K H \int_{0}^{D} \bar{\rho}(D-\sigma) \partial_{t} u_{\mathrm{av}}(\sigma, t) d \sigma\right],
\end{aligned}
$$

onde o filtro passa baixa é representado na forma de espaço de estado.

\section{Passo 2: Transformação backstepping no sistema alvo}

Utilizando a notação, usamos $w(x, t)$ para denotar o estado médio transformado. De (28), a transformação backstepping

$$
\begin{aligned}
w(x, t)=u_{\mathrm{av}}(x, t) & \\
& -\int_{0}^{x} \gamma(x-\sigma) \partial_{t} u_{\mathrm{av}}(\sigma, t) d \sigma-\gamma(x) \vartheta_{\mathrm{av}}(t)
\end{aligned}
$$

mapeia o sistema em malha fechada médio (49)-(52) no sistema alvo exponencialmente estável (mostrado no Passo 3 )

$$
\begin{aligned}
& \dot{\vartheta}_{\mathrm{av}}(t)=K H \vartheta_{\mathrm{av}}(t)+w_{x}(0, t), \\
& \partial_{t t} w(x, t)=\partial_{x x} w(x, t), \quad x \in[0, D], \\
& w(0, t)=0, \\
& w_{t}(D, t)=-\frac{1}{\bar{c}} \partial_{x} w(D, t), \quad \bar{c}>0, \\
& \partial_{x} w(D, t)=-\frac{1}{c} \partial_{t} \partial_{x} u_{\mathrm{av}}(D, t) .
\end{aligned}
$$

O sistema alvo (54)-(58) pode ser derivado fazendo uso da transforção backstepping inversa (veja Susto and Krstić (2010))

$$
\begin{array}{rl}
u_{\mathrm{av}}(x, t)=w(x, t)+K H & n(x) \vartheta_{\mathrm{av}}(t) \\
& +K H \int_{0}^{x} n(x-\sigma) w_{t}(\sigma, t) d \sigma,
\end{array}
$$

com

$$
n(x)=\left[\begin{array}{ll}
0 & I
\end{array}\right] e^{\bar{A} x}\left[\begin{array}{ll}
I & 0
\end{array}\right]^{T}, \quad \bar{A}=\left(\begin{array}{cc}
0 & (K H)^{2} \\
I & 0
\end{array}\right),
$$

no sistema em malha fechada médio (49)-(52). Além disso, tomando a derivada no tempo da transformação backstepping (53) junto com (52) e sua inversa (59), chegamos em (58) lembrando que $\dot{U}_{\text {av }}(t)=\partial_{t} \partial_{x} u_{\text {av }}(D, t)$, ou equivalentemente,

$$
\begin{aligned}
& \partial_{t} w_{x}(D, t)=-c w_{x}(D, t)+K H w(D, t) \\
& +(K H)^{2} n(D) \vartheta_{\mathrm{av}}(t)+(K H)^{2} \int_{0}^{D} n(D-\sigma) w_{t}(\sigma, t) d \sigma .
\end{aligned}
$$

\section{Passo 3: Estabilidade exponencial do sistema de alvo}

Começamos introduzindo as normas do sistema

$$
\begin{aligned}
& \Omega(t)=\left\|\partial_{x} u_{\mathrm{av}}(t)\right\|^{2}+\left\|\partial_{t} u_{\mathrm{av}}(t)\right\|^{2}+\left|\vartheta_{\mathrm{av}}(t)\right|^{2}+\left|\partial_{x} u_{\mathrm{av}}(D, t)\right|^{2}, \\
& \Xi(t)=\left\|w_{x}(t)\right\|^{2}+\left\|w_{t}(t)\right\|^{2}+\left|\vartheta_{\mathrm{av}}(t)\right|^{2}+\left|w_{x}(D, t)\right|^{2} .
\end{aligned}
$$

Para comprovar a estabilidade do sistema em malha fechada, consideramos o funcional de Lyapunov-Krasovskii

$$
V(t)=\frac{\vartheta_{\mathrm{av}}^{2}(t)}{2}+a E(t)+\frac{b}{2} w_{x}^{2}(D, t)
$$

onde os parâmetros $a, b>0$ são escolhidos mais tarde e o funcional $E(t)$ é definido por (Susto and Krstić (2010)): 


$$
\begin{aligned}
E(t)= & \frac{1}{2}\left(\left\|w_{x}(t)\right\|^{2}+\left\|w_{t}(t)\right\|^{2}\right)+ \\
& +\delta \int_{0}^{D}(1+y) w_{x}(y, t) w_{t}(y, t) d y,
\end{aligned}
$$

onde $\delta>0$ também é um parâmetro a ser escolhido, posteriormente. Observamos que

$$
\theta_{1} \Xi \leq V \leq \theta_{2} \Xi
$$

onde

$$
\begin{aligned}
& \theta_{1}=\min \left\{\frac{1}{2}, \frac{a}{2}[1-\delta(1+D)], \frac{b}{2}\right\}, \\
& \theta_{2}=\min \left\{\frac{1}{2}, \frac{a}{2}[1+\delta(1+D)], \frac{b}{2}\right\} .
\end{aligned}
$$

Escolhemos

$$
0<\delta<\frac{1}{1+D},
$$

a fim de garantir que $\theta_{1}$ e $\theta_{2}$ são não-negativos e, portanto, a função Lyapunov $V$ em (64) é positiva definida. Em seguida, calculamos a derivada temporal de $E(t)$

$$
\begin{aligned}
\dot{E}(t)= & -\frac{\delta}{2}\left[\left\|w_{x}(t)\right\|^{2}+\left\|w_{t}(t)\right\|^{2}+w_{x}(0, t)^{2}\right]+ \\
& +\frac{\delta}{2}(1+D)\left[w_{t}(D, t)^{2}+w_{x}(D, t)^{2}\right]+ \\
& +w_{x}(D, t) w_{t}(D, t) .
\end{aligned}
$$

De (57), substituímos a condição de contorno $w_{x}(D, t)=$ $-\bar{c} w_{t}(D, t)$ e obtemos

$$
\begin{aligned}
\dot{E}(t)= & -\frac{\delta}{2}\left[\left\|w_{x}(t)\right\|^{2}+\left\|w_{t}(t)\right\|^{2}+w_{x}(0, t)^{2}\right]+ \\
& -\left[\bar{c}-\delta \frac{1+D}{2}\left(1+\bar{c}^{2}\right)\right] w_{t}(D, t)^{2} .
\end{aligned}
$$

Escolhendo agora

$$
\delta<\frac{2 \bar{c}}{(1+D)\left(1+\bar{c}^{2}\right)},
$$

temos que a constante entre parênteses no segundo termo de (71) é positiva. Agora, calculando a derivada completa de $V(t)$, associado à solução do sistema alvo (54)-(58), temos:

$$
\begin{aligned}
\dot{V}(t) & =K H \vartheta_{\mathrm{av}}^{2}(t)+\vartheta_{\mathrm{av}}(t) w_{x}(0, t) \\
& +a \dot{E}(t)+b w_{x}(D, t) \partial_{t} w_{x}(D, t) .
\end{aligned}
$$

Ao aplicar a desigualdade de Young ao segundo termo em (73), podemos escrever

$$
\begin{aligned}
\dot{V}(t) & \leq \frac{K H}{2} \vartheta_{\mathrm{av}}^{2}(t)+\left[\frac{1}{2|K H|}-a \frac{\delta}{2}\right] w_{x}(0, t)^{2}+ \\
& -a \frac{\delta}{2}\left[\left\|w_{x}(t)\right\|^{2}+\left\|w_{t}(t)\right\|^{2}\right]+b w_{x}(D, t) \partial_{t} w_{x}(D, t) .
\end{aligned}
$$

Escolhendo

$$
a \geq \frac{1}{\delta|K H|}
$$

obtemos agora

$$
\begin{aligned}
\dot{V}(t) & \leq \frac{K H}{2} \vartheta_{\mathrm{av}}^{2}(t)-a \frac{\delta}{2}\left[\left\|w_{x}(t)\right\|^{2}+\left\|w_{t}(t)\right\|^{2}\right] \\
& +b w_{x}(D, t) \partial_{t} w_{x}(D, t) .
\end{aligned}
$$

Finalmente, substituindo (61) em (76), o último termo no lado direito pode ser tratado analogamente ao que foi feito nas referências (Feiling et al. (2018)) e (Oliveira et al. (2018)) para o processo de difusão para processos de difusão ou mesmo realizados em (Oliveira et al. (2017)) com atrasos puros. Depois de longos cálculos, aplicando as desigualdades de Young, Poincaré, Agmon e Cauchy-Schwarz (mais de uma) e a ajuda da integração por partes, concluímos que existe $c^{*}>0$ (dependendo de $K H$ e $D)$ e $\eta>0$ tal que, para $c>c^{*}$ suficientemente grande em (40), temos $\dot{V}(t) \leq-\eta V(t)$ e o sistema alvo (54)-(58) é exponencialmente estável na norma
$\left(\left|\vartheta_{\mathrm{av}}(t)\right|^{2}+\left\|w_{x}(t)\right\|^{2}+\left\|w_{t}(t)\right\|^{2}+\left|w_{x}(D, t)\right|^{2}\right)^{1 / 2}$, ou seja, nas variáveis transformadas $\left(\vartheta_{\mathrm{av}}, w\right)$.

Passo 4: Estimativa da estabilidade exponencial $\left(\mathscr{H}_{1}\right)$ do sistema em malha fechada médio

Na última etapa, chegamos à estimativa

$$
V(t) \leq e^{-\eta t} V(0) \text {. }
$$

Para provar a estabilidade do sistema em malha fechada em suas variáveis originais $\left(\vartheta_{\mathrm{av}}, u_{\mathrm{av}}\right)$ de (77) fornecemos desigualdades relacionando as variáveis $u(x, t)$ e $w(x, t)$. Da transformação inversa (59) obtemos que

$$
\begin{aligned}
\partial_{x} u_{\mathrm{av}}(x, t)= & w_{x}(x, t)+\int_{0}^{x} \phi^{\prime}(x-y) w(y, t) d y+ \\
& +\int_{0}^{x} n^{\prime}(x-y) w_{t}(y, t) d y+\psi(x)^{\prime} \vartheta_{\mathrm{av}}(t), \\
\partial_{t} u_{\mathrm{av}}(x, t)= & w_{t}(x, t)+\int_{0}^{x} \phi(x-y) w_{t}(y, t) d y+ \\
& +\int_{0}^{x} n^{\prime}(x-y) w(y, t) d y+\psi(x) K H \vartheta_{\mathrm{av}}(t) .
\end{aligned}
$$

Aplicando as desigualdades de Poincaré, Young e CauchySchwarz, obtemos

$$
\begin{aligned}
& \left\|\partial_{x} u_{\mathrm{av}}(t)\right\|^{2} \leq \alpha_{1}\left\|w_{x}(t)\right\|^{2}+\alpha_{2}\left\|w_{t}(t)\right\|^{2}+\alpha_{3}\left|\vartheta_{\mathrm{av}}(t)\right|^{2} \\
& \left\|\partial_{t} u_{\mathrm{av}}(t)\right\|^{2} \leq \beta_{1}\left\|w_{x}(t)\right\|^{2}+\beta_{2}\left\|w_{t}(t)\right\|^{2}+\beta_{3}\left|\vartheta_{\mathrm{av}}(t)\right|^{2}
\end{aligned}
$$

onde

$$
\begin{aligned}
& \alpha_{1}=4\left(1+4 D^{3}\left\|\phi^{\prime}\right\|^{2}\right), \\
& \alpha_{2}=4 D\left\|n^{\prime}\right\|^{2}, \\
& \alpha_{3}=4\left\|\psi^{\prime}\right\|^{2}, \\
& \beta_{1}=4\left\|n^{\prime}\right\|^{2} \\
& \beta_{2}=4\left(1+4 D^{3}\|\phi\|^{2}\right), \\
& \beta_{3}=4\|\psi K H\|^{2} .
\end{aligned}
$$

Aplicando (80), obtemos

$$
\Omega(t) \leq \theta_{4} \Xi(t),
$$

onde

$$
\theta_{4}=\max \left\{\alpha_{1}+\beta_{1}, \alpha_{2}+\beta_{2}, \alpha_{3}+\beta_{3}\right\} .
$$

Com ajuda das derivadas no tempo e espaço de (28) - veja (Susto and Krstić (2010)) para mais detalhes - e aplicando novamente as desigualdades de Poincaré, Young e CauchySchwarz, obtemos as seguintes desigualdades:

$$
\begin{aligned}
& \left\|w_{x}(t)\right\|^{2} \leq a_{1}\left\|\partial_{x} u_{\mathrm{av}}(t)\right\|^{2}+a_{2}\left\|\partial_{t} u_{\mathrm{av}}(t)\right\|^{2}+a_{3}\left|\vartheta_{\mathrm{av}}(t)\right|^{2}, \\
& \left\|w_{t}(t)\right\|^{2} \leq b_{1}\left\|\partial_{x} u_{\mathrm{av}}(t)\right\|^{2}+b_{2}\left\|\partial_{t} u_{\mathrm{av}}(t)\right\|^{2}+b_{3}\left|\vartheta_{\mathrm{av}}(t)\right|^{2},
\end{aligned}
$$

por meio destas, obtemos

$$
\theta_{3} \Xi \leq \Omega(t)
$$

onde

$$
\theta_{3}=\frac{1}{\max \left\{a_{1}+b_{1}, a_{2}+b_{2}, a_{3}+b_{3}+1\right\}} .
$$

Com a ajuda de (66), (77), (87) e (91), chegamos em

$$
\Omega(t) \leq \frac{\theta_{1} \theta_{3}}{\theta_{2} \theta_{4}} \Omega(0) e^{-\eta t},
$$

que completa a prova da estabilidade exponencial do sistema médio em malha fechada no sentido da norma (62) nas variáveis $\left(\vartheta_{\mathrm{av}}, u_{\mathrm{av}}\right)$.

Passos 5 e 6: Devido a limitações de espaço, não podemos entrar em detalhes no Passo 5 (Teorema da Média) e no Passo 6 (convergência ao extremo), mas a demonstração é semelhante às apresentadas em (Oliveira et al. (2017)) e (Feiling et al. (2018)). De acordo com (93), a origem do sistema em malha fechada médio com EDP da onda é exponencialmente estável. 
Aplicando o teorema da média para sistemas de dimensão infinita desenvolvidos em ((Hale and Lunel, 1990, Section 2)), para $\omega$ suficientemente grande, (49)-(52) tem uma solução periódica exponencialmente estável em torno de seu equilíbrio satisfazendo (45). Juntamente com (45), não é difícil mostrar

$$
\limsup _{t \rightarrow \infty}|\tilde{\theta}(t)|=\mathscr{O}(1 / \omega)
$$

De (10) e da Figura 1, podemos escrever $\theta(t)-\theta^{*}=\tilde{\theta}(t)+$ $S(t)$, e recordando que $S(t)$ em (24) é da ordem $\mathscr{O}(a / \omega)$, finalmente chegamos a (46). A convergência do atuador propagado $\Theta(t)$ para o otimizador $\Theta^{*}$ é mais fácil de se provar. Usando (25) e tomando o seu valor absoluto, tem-se

$$
\left|\Theta(t)-\Theta^{*}\right|=|\vartheta(t)+a \sin (\omega(t))| .
$$

Assim como na prova de convergência de $\theta(t)$ ao seu valor de entrada ótimo $\theta^{*}$ discutida acima, escrevemos (95) em termos da solução periódica $\vartheta^{\Pi}(t)$ e seguimos os mesmos passos aplicando-se a desigualdade de Young e o resultado que $\vartheta(t)-\vartheta^{\Pi}(t) \rightarrow 0$ exponencialmente através do teorema da média ((Hale and Lunel, 1990, Section 2)). Assim, de (45), chegamos a (47). Para mostrar a convergência da saída $y(t)$ do mapeamento estático para o valor ideal $y^{*}$, substituímos $\Theta(t)-$ $\Theta^{*}$ em (9) por (25) e tomamos o valor absoluto

$$
\left|y(t)-y^{*}\right|=\left|\frac{H}{2}[\vartheta(t)+a \sin (\omega(t))]^{2}\right| .
$$

Expandindo o termo quadrático de (96) e aplicando a desigualdade de Young à equação resultante, tem-se $\left|y(t)-y^{*}\right|=$ $\left|H\left[\vartheta(t)^{2}+a^{2} \sin ^{2}(\omega t)\right]\right|$. Como antes, adicionamos a solução periódica $\vartheta^{\Pi}(t)$, aplicamos a desigualdade de Young e a relação $\vartheta(t)-\vartheta^{\Pi}(t) \rightarrow 0$ do teorema da média ((Hale and Lunel, 1990, Section 2)). Assim, novamente com (45), temos (48).

\section{SIMULAÇÕES NUMÉRICAS}

Considere um mapeamento estático quadrático como em (8), com Hessiana $H=-0.2$, otimizador ideal $\Theta^{*}=2$, e valor ótimo $y^{*}=5$. O comprimento do domínio da EDP da onda é estabelecido para $D=1$. Os parâmetros da busca extremal proposta são escolhidos como $\omega=10, a=0.2, c=10, \bar{c}=0.5 \mathrm{e}$ $K=0.4$. O resultado da simulação em malha fechada é ilustrado na Figura 2. Observamos que a variável $\Theta$ converge para uma vizinhança de $\Theta^{*}$ ótimo, como esperado.

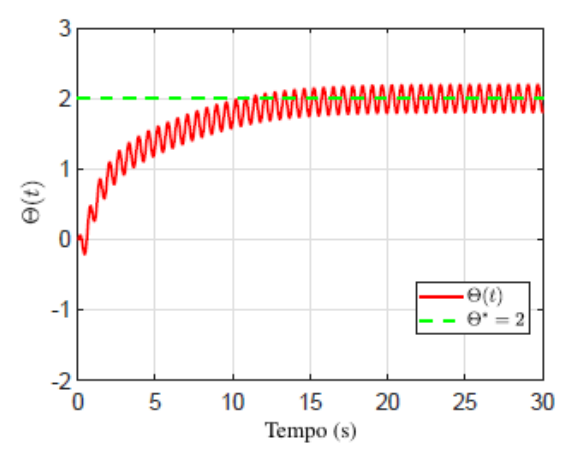

(a) Parâmetro $\Theta(t)$ convergindo para $\Theta^{*}$.

Figura 2. Controle extremal baseado em gradiente com EDP da onda na dinâmica de atuação.

\section{CONCLUSÃO}

Este trabalho forneceu uma análise geral abordando-se os pontos principais da aplicação de projetos de busca extremal para compensação de EDPs da onda. Foi apresentado o método de busca extremal por gradiente para mapeamentos estáticos escalares com dinâmica de atuação governada por EDPs de onda. A dinâmica de atuação deve ser conhecida, mas nenhum conhecimento é assumido para os parâmetros do mapeamento. A lei de controle média para compensar a dinâmica de atuação das ondas foi construída combinando técnicas de análise de ponta na área de controle de fronteira de equações diferenciais parciais via metodologia de backstepping e empregando perturbações do gradiente e da Hessiana baseadas em estimativas do mapeamento estático. $\mathrm{O}$ sinal aditivo de perturbação também levou em consideração a dinâmica de onda e a compensou. Por fim, a estabilidade exponencial local e a convergência para uma pequena vizinhança do extremo desejado foram garantidas. A abordagem proposta também tem ampla aplicabilidade na prática, uma vez que a presença de modelos de EDP são frequentemente listados como um grande fator limitante na aplicação de controladores de busca extremal em algumas situações práticas.

\section{REFERÊNCIAS}

Aarsnes, U.J.F., Aamo, O.M., and Krstić, M. (2019). Extremum seeking for real-time optimal drilling control. American Control Conference, 5222-5227.

Bekiaris-Liberis, N. and Krstić, M. (2014). Compensation of wave actuator dynamics for nonlinear systems. IEEE Transactions on Automatic Control, 59(6), 1555-1570.

Feiling, J., Koga, S., Krstić, M., and Oliveira, T.R. (2018). Gradient extremum seeking for static maps with actuation dynamics governed by diffusion PDEs. Automatica, 95(9), 197-206.

Ghaffari, A., Krstić, M., and Nešić, D. (2012). Multivariable Newton-based extremum seeking. Automatica, 48(8), 17591767.

Hale, J.K. and Lunel, S.V. (1990). Averaging in infinite dimensions. J. Integral Equations Appl, 2(4), 463-494.

Khalil, H.K. (1996). Noninear Systems. Prentice-Hall, New Jersey.

Krstić, M. (2009). Compensating a string PDE in the actuation or sensing path of an unstable ODE. IEEE Transactions on Automatic Control, 54(6), 1362-1368.

Krstić, M. and Smyshlyaev, A. (2008). Boundary control of PDEs: A course on backstepping designs, volume 16. Siam.

Krstić, M. and Wang, H.H. (2000). Stability of extremum seeking feedback for general nonlinear dynamic systems. Automatica, 36(4), 595-601.

Oliveira, T.R., Feiling, J., Koga, S., and Krstić, M. (2018). Scalar Newton-based extremum seeking for a class of diffusion PDEs. IEEE Conference on Decision and Control, 29262931.

Oliveira, T.R., Krstic, M., and Tsubakino, D. (2017). Extremum seeking for static maps with delays. IEEE Transactions on Automatic Control, 62(4), 1911-1926.

Rusiti, D., Oliveira, T.R., Evangelisti, G., Gerdts, M., and Krstić, M. (2019). Stochastic extremum seeking for dynamic maps with delays. IEEE Control Systems Letters, 3(1), 6166.

Rusiti, D., Oliveira, T.R., Mills, G., and Krstić, M. (2018). Newton-based extremum seeking for higher derivatives of unknown maps with delays. European Journal of Control, 42(5), 72-83.

Susto, G.A. and Krstić, M. (2010). Control of PDE-ODE cascades with Neumann interconnections. Journal of the Franklin Institute, 347(1), 284-314. 